\title{
Une évaluation qualitative du programme de la Base de données publique internationale sur les éclosions des maladies d'origine alimentaire
}

\author{
Abhinand Thaivalappil1*, Mariola Mascarenhas ${ }^{2}$, Lisa A Waddell², lan Young ${ }^{3}$
}

\section{Résumé}

Contexte : La Base de données publique internationale sur les éclosions des maladies d'origine alimentaire (Publicly Available International Foodborne Outbreak Database (PAIFOD)) est un répertoire régulièrement mis à jour qui contient des données internationales sur les éclosions recueillies auprès de multiples systèmes de surveillance et de sources. En date de février 2020, la base de données contenait plus de 13000 entrées couvrant plus de 20 ans. La PAIFOD est la seule base de données connue qui saisit les données internationales sur les éclosions de maladies d'origine alimentaire.

Objectif : Explorer les perceptions des utilisateurs et déterminer les orientations possibles pour la PAIFOD et faire des recommandations pour les bases de données contenant des informations sur la sécurité alimentaire.

Méthodes : Entre janvier et mars 2020, 16 entretiens téléphoniques semi-directifs ont été menés avec 24 anciens utilisateurs, actuels et potentiels de la PAIFOD. Les répondants ont été interrogés sur leur connaissance et leur expérience de l'utilisation de la PAIFOD ainsi que sur ses forces et ses faiblesses, et sur les recommandations concernant la base de données. Une approche d'analyse thématique inductive a été utilisée pour analyser les données qualitatives et générer des thèmes.

Résultats : Quatre thèmes principaux ont été dégagés à partir des témoignages des 24 répondants sur leur expérience et leurs recommandations concernant la PAIFOD : les participants ont considéré que la PAIFOD était un outil utile; ils ne connaissaient pas bien son contenu ni son objectif; ils ont déclaré qu'elle devrait devenir une plateforme en libre accès ou être liée à une autre initiative de partage d'informations; et ils ont estimé que la PAIFOD avait le potentiel pour améliorer la réputation de l'Agence en devenant largement reconnue et utilisée.

Conclusion : Ce travail, ainsi que le contexte en constante évolution de la surveillance alimentaire, soutient la nécessité de garantir que la PAIFOD soit mise à jour pour répondre aux exigences actuelles des experts en salubrité alimentaire.
Cette oeuvre est mise à la disposition selon les termes de la licence internationale Creative Commons Attribution 4.0

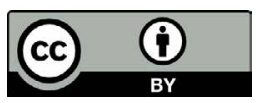

Affiliations

1 Department of Population Medicine, University of Guelph, Guelph, ON

${ }^{2}$ Laboratoire national de microbiologie, Agence de la santé publique du Canada, Guelph, ON

${ }^{3}$ School of Occupational and Public Health, Ryerson University, Toronto, ON

\section{*Correspondance :}

athaival@uoguelph.ca

Citation proposée : Thaivalappil A, Mascarenhas M, Waddell LA, Young I. Une évaluation qualitative du programme de la Base de données publique internationale sur les éclosions des maladies d'origine alimentaire. Relevé des maladies transmissibles au Canada 2021;47(1):68-74. https://doi.org/10.14745/ccdr.v47i01a09f Mots-clés : base de données, évaluation, PAIFOD, éclosions de maladie d'origine alimentaire, analyse thématique, recherche qualitative

\section{Introduction}

La communication des données relatives aux éclosions de maladie d'origine alimentaire est importante pour évaluer les enseignements tirés, déterminer les tendances et les modèles et orienter les futures politiques de santé publique, les évaluations des risques et les stratégies d'atténuation (1).
En 2000, la Division des sciences des risques pour la santé publique du Laboratoire national de microbiologie de l'Agence de la santé publique du Canada (l'Agence) a lancé la Base de données publique internationale sur les éclosions des maladies d'origine alimentaire (PAIFOD). La PAIFOD est un répertoire de 
Figure 1 : Un aperçu de la Base de données publique internationale sur les éclosions des maladies d'origine alimentaire, de ses colonnes et des caractéristiques des éclosions qui y sont saisies

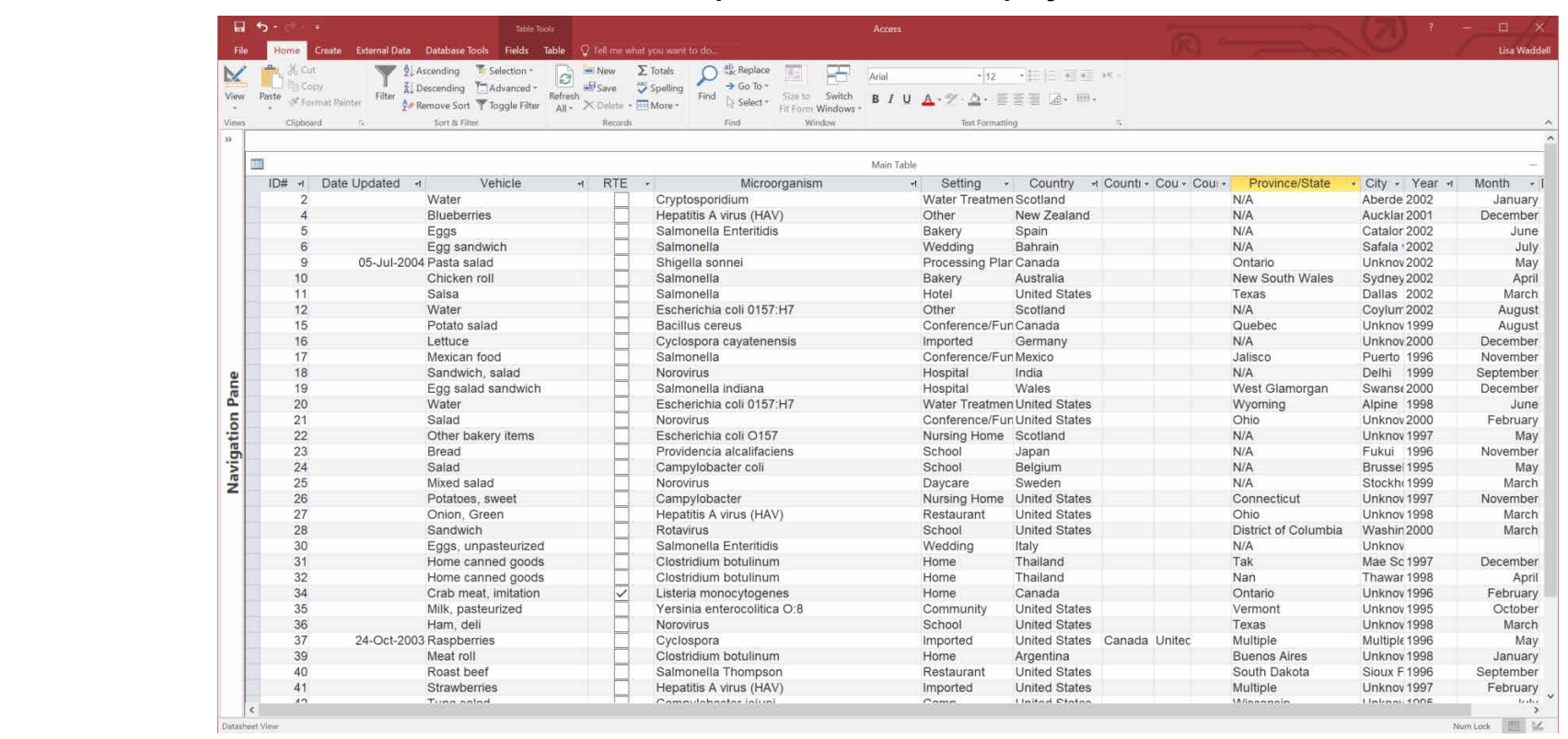

Abbreviation: RTE, ready to eat

Note : La base de données est hébergée sur Microsoft Access et seuls quelques champs sont affichés

données internationales sur les éclosions de maladies d'origine alimentaire enregistrées par divers systèmes de surveillance et sources publics, tels que des rapports, des listes de diffusion, des communiqués de presse, des sites Web gouvernementaux et des revues avec comité de lecture. À ce jour, la PAIFOD est la seule base de données connue permettant de saisir des informations sur les épidémies mondiales d'origine alimentaire.

Les universités et les clients des gouvernements fédéral, provinciaux et territoriaux utilisent les informations de la PAIFOD pour établir les dossiers de preuve, les résumés des risques, les évaluations des risques, les analyses des épidémies et d'autres projets de recherche (2-5). La PAIFOD utilise Microsoft Access (Redmond, Washington, États-Unis) pour sauvegarder les données relatives aux éclosions (figure 1). En date de février 2020, la base de données contenait 13355 entrées. Les éclosions enregistrées datent de 1945 à nos jours. Actuellement, la PAIFOD contient des informations sur 31 espèces bactériennes, 20 parasites, 9 virus, 7 biotoxines marines et 3 mycotoxines. Les éclosions d'origine alimentaire les plus fréquemment détectées sont liées à Salmonella Enteritidis ( $n=2420$ ) et aux norovirus ( $n=1$ 958).

La PAIFOD est mise à jour quotidiennement. En moyenne, cinq éclosions sont ajoutées chaque semaine à la base de données, avec des variations saisonnières. Un résumé des champs contenus dans la PAIFOD est présenté dans le tableau 1. La base de données n'est pas accessible au public. II faut plutôt demander des rapports de synthèse personnalisés en contactant le gestionnaire de la base de données, à l'Agence (voir Remerciements).
Depuis ses débuts, la PAIFOD n'a cessé de croître en taille et en fréquence d'utilisation. Cependant, une évaluation des besoins des intervenants n'a jamais été réalisée pour évaluer la base de données et cibler les possibilités d'amélioration.

Les auteurs ont procédé à une évaluation qualitative du programme afin d'obtenir les commentaires des intervenants sur la base de données et de mesurer l'intérêt pour une variété de modifications possibles de la PAIFOD. L'objectif de cette étude était d'explorer les perceptions des utilisateurs sur la base de données, d'évaluer ses forces, ses faiblesses et les domaines à améliorer.

\section{Méthodes}

\section{Participants à l'étude}

Des entretiens semi-directifs ont été menés avec des utilisateurs anciens, actuels ou futurs de la PAIFOD entre janvier et mars 2020. À partir des utilisateurs et des réseaux de la PAIFOD, une liste de 47 personnes, issues de 29 ministères et divisions différents, a été établie aux fins de contacts et de recrutement. Pour les besoins des entretiens et de l'analyse, chaque ministère ou division unique en tant qu'étude distincte a été considéré comme un " participant » et une unité d'analyse. Les participants étaient membres des ministères et divisions des gouvernements fédéral, provinciaux et municipaux, ainsi que des chercheurs universitaires. Les participants ont été recrutés par courrier électronique pour un entretien individuel ou de groupe, en fonction du nombre de personnes au sein de chaque ministère ou division. 
Tableau 1 : Tous les champs saisis dans la Base de données publique internationale sur les éclosions des maladies d'origine alimentaireet leur description

\begin{tabular}{|c|c|}
\hline Catégorie & Domaine(s) \\
\hline $\begin{array}{l}\text { Produit } \\
\text { alimentaire }\end{array}$ & $\begin{array}{l}\text { - Véhicule } \\
\text { - Prêt-à-manger (si le produit alimentaire était prêt } \\
\text { à être consommé) }\end{array}$ \\
\hline $\begin{array}{l}\text { Micro- } \\
\text { organisme }\end{array}$ & $\begin{array}{l}\text { Virus, espèces bactériennes, champignons ou } \\
\text { parasites responsables précis }\end{array}$ \\
\hline Géographie & $\begin{array}{l}\text { - Pays } \\
\text { - Province ou état } \\
\text { - Ville } \\
\text { - Cadre (e.g. école, restaurant) }\end{array}$ \\
\hline Date & $\begin{array}{l}\text { - Année } \\
\text { - Mois } \\
\text { - Jour }\end{array}$ \\
\hline $\begin{array}{l}\text { Informations sur } \\
\text { les cas }\end{array}$ & $\begin{array}{l}\text { - Cas suspects } \\
\text { - Cas confirmés } \\
\text { - Groupe(s) d'âge } \\
\text { - Nombre d'hospitalisations } \\
\text { - Nombre de décès } \\
\text { - Symptômes } \\
\text { - Principales séquelles si elles sont signalées }\end{array}$ \\
\hline $\begin{array}{l}\text { Renseignements } \\
\text { supplémentaires }\end{array}$ & $\begin{array}{l}\text { - Cause (e.g., rupture dans la chaîne du froid, } \\
\text { consommation d'aliments crus) } \\
\text { - Concentration (e.g. CFU/ml) } \\
\text { - Vérification (oui/non) } \\
\text { - Confirmation (en laboratoire, } \\
\text { épidémiologiquement }\end{array}$ \\
\hline Autres & $\begin{array}{l}\text { - Source (e.g., détails de l'article de journal, nom du } \\
\text { journal) } \\
\text { - Informations sensibles (oui/non) } \\
\text { - URL du site Web } \\
\text { - Histoire (i.e. description écrite des informations } \\
\text { pertinentes extraites du document source) }\end{array}$ \\
\hline
\end{tabular}

Abréviations : CFU, unités formant des colonies; URL, Uniform Resource Locator (adresse URL)

Cette étude n'a pas été examinée par le comité d'éthique de la recherche de l'université Ryerson, car elle est classée comme une évaluation de programme (6).

\section{Collecte de données}

Les participants ont été questionnés par téléphone à l'aide d'un guide d'entretien semi-directif. Les questions de l'entretien étaient ouvertes et portaient sur (a) les connaissances des utilisateurs de la PAIFOD; (b) l'expérience des utilisateurs avec la PAIFOD; (c) les forces et les faiblesses de la base de données; et (d) les recommandations d'amélioration. Le guide d'entretien (disponible sur demande auprès des auteurs) a été modifié en fonction de l'expérience des participants avec la base de données.

Les entretiens duraient entre 15 et 50 minutes, et le nombre de participants variait entre un et quatre. Les entretiens ont été enregistrés sur support audio pour en garantir l'exactitude. Dans un cas, l'intervieweur a écrit ses notes après l'entretien parce qu'il avait des difficultés techniques avec le dispositif d'enregistrement. Les enregistrements ont été transcrits par des professionnels, et les transcriptions ont été validées et anonymisées avant d'être analysées.

Les noms utilisés dans cet article sont des pseudonymes aléatoires utilisés aux fins de confidentialité.

Des méthodes de validation ont été utilisées : deux enquêteurs ont analysé et interprété les données obtenues pour ajouter de multiples perspectives, et des entretiens individuels et de groupe approfondis ont été menés (7). Des contrôles ont été effectués par les membres afin d'accroître la validité des résultats (8).

\section{Analyse des données}

L'équipe de recherche a analysé les données en utilisant une approche d'analyse thématique inductive dans un cadre constructionniste (9). II s'agissait d'un processus de création de catégories basé sur des données (10). Le processus de codage comprenait des lectures répétées des transcriptions afin de cerner les tendances, les incohérences et les contradictions entre les données. Deux enquêteurs ont examiné cinq transcriptions de façon indépendante et ont généré une liste de codes. Le cadre de codage a été consolidé et affiné au cours de la discussion. Les autres transcriptions ont également été codées individuellement, puis consolidées. Les thèmes ont été générés en utilisant une approche latente, c'est-à-dire en examinant les hypothèses, les idées et les significations et en ciblant les thèmes basés sur des interprétations du contenu des entretiens (10). Les thèmes ont été établis, révisés, modifiés, définis et nommés. Des extraits de données (i.e. des citations) ont été sélectionnés pour représenter au mieux chaque thème. L'analyse a été réalisée à l'aide du logiciel d'analyse qualitative NVivo 12 (OSR International, Doncaster, Australie).

\section{Résultats}

Au total, 16 entretiens ont été menés avec 24 personnes. La plupart des participants provenaient de différents ministères et divisions de l'Agence $(n=8,33 \%)$, de l'Agence canadienne d'inspection des aliments (ACIA; $n=5,21 \%$ ) et de Santé Canada $(n=5,21 \%)$ (tableau 2). La plupart des répondants étaient des utilisateurs anciens ou actuels de la PAIFOD $(n=15,63 \%)$.

Quatre thèmes comportant chacun trois ou quatre sous-thèmes ont été générés à partir du cadre de codage $(n=29)$. Les thèmes sont présentés ci-dessous, les commentaires des participants étant cités textuellement à titre d'illustration.

\section{Un outil utile qui guide le travail des experts} Les demandes sont adaptées et opportunes. Les participants qui avaient utilisé la PAIFOD $(n=16)$ ont rapidement mentionné l'importance de cette ressource pour leur travail. Ils ont trouvé que le service et la communication étaient rapides. Par exemple :

Hannah : Elles ont été très utiles et faciles à obtenir, le personnel de l'Agence est très bien informé et très rapide et, oui, très 
Tableau 2 : Renseignements sur les participants aux entretiens

\begin{tabular}{|c|c|c|c|c|}
\hline $\begin{array}{c}\text { Identification } \\
\text { du } \\
\text { participant }\end{array}$ & Entretien & Pseudonymes & Organisation $^{a}$ & $\begin{array}{l}\text { Statut d'utilisateur } \\
\text { de la PAIFOD }\end{array}$ \\
\hline 1 & $A$ & Dimitri & Agence canadienne d'inspection des aliments & Ancientérieur/actuel \\
\hline 2 & B & Hannah & Agence canadienne d'inspection des aliments & Ancientérieur/actuel \\
\hline 3 & C & Susan & Université de Guelph & Jamais utilisé \\
\hline 4 & D & Todd & Agence canadienne d'inspection des aliments & Ancientérieur/actuel \\
\hline 5 & $E$ & Marie & Université de Guelph & Ancien utilisateur \\
\hline 6 & $\mathrm{~F}$ & Anna & Agence de la santé publique du Canada & Ancientérieur/actuel \\
\hline 7 & $\mathrm{~F}$ & Kate & Agence de la santé publique du Canada & Ancientérieur/actuel \\
\hline 8 & $\mathrm{~F}$ & Richard & Agence de la santé publique du Canada & Ancientérieur/actuel \\
\hline 9 & G & Rachel & Agence de la santé publique du Canada & Ancientérieur/actuel \\
\hline 10 & G & Shelly & Agence de la santé publique du Canada & Jamais utilisé \\
\hline 11 & G & Luc & Agence de la santé publique du Canada & Ancientérieur/actuel \\
\hline 12 & G & Rebecca & Agence de la santé publique du Canada & Jamais utilisé \\
\hline 13 & $\mathrm{H}$ & Rose & Santé Canada & Ancientérieur/actuel \\
\hline 14 & $\mathrm{H}$ & Leila & Santé Canada & Ancientérieur/actuel \\
\hline 15 & I & Aaron & Santé Canada & Jamais utilisé \\
\hline 16 & J & Yen & Santé publique Ontario & Ancientérieur/actuel \\
\hline 17 & $\mathrm{~J}$ & Manon & Santé publique Ontario & Ancientérieur/actuel \\
\hline 18 & K & Olivia & Agence canadienne d'inspection des aliments & Jamais utilisé \\
\hline 19 & L & Joon & $\begin{array}{l}\text { Ministère de l'Agriculture, de l'Alimentation et des Affaires rurales de } \\
\text { l'Ontario }\end{array}$ & Jamais utilisé \\
\hline 20 & L & Chris & $\begin{array}{l}\text { Ministère de l'Agriculture, de l'Alimentation et des Affaires rurales de } \\
\text { I'Ontario }\end{array}$ & Jamais utilisé \\
\hline 21 & M & Kim & Santé Canada & Ancientérieur/actuel \\
\hline 22 & $\mathrm{~N}$ & Moshe & Santé Canada & Ancientérieur/actuel \\
\hline 23 & $\mathrm{O}$ & Farid & Agence canadienne d'inspection des aliments & Jamais utilisé \\
\hline 24 & $\mathrm{P}$ & Mark & Agence de la santé publique du Canada & Jamais utilisé \\
\hline
\end{tabular}

Abréviations : PAIFOD, Base de données publique internationale sur les éclosions des maladies d'origine alimentaire

a Les divisions au sein des organisations sont omises pour des raisons de confidentialité

impressionnant, un programme très impressionnant et un produit utile.

Les rapports sont détaillés, répondent aux besoins et aux attentes. Les utilisateurs ont généralement trouvé que les rapports étaient adaptés à leurs besoins, souvent à la suite de leurs conversations avec le gestionnaire de la base de données.

Todd : J'ai été très satisfait de la réactivité et du délai d'exécution qui nous sont accordés lorsque nous demandons des informations et je trouve qu'ils sont très bons pour toute clarification ou précision de nos demandes; on pourrait insister sur le fait que cela est fait en temps opportun.

Une relation personnelle avec le gestionnaire de la base de données. Les clients ont mentionné leur relation avec les gestionnaires de base de données précédents et actuels qui ont aidé à générer les résultats requis. Par exemple :
Rose : Et parfois, ils ajoutent un élément à notre recherche. Ils diront, " j'ai regardé dans la base de données, je n'ai rien trouvé, mais rapidement voici mon opinion sur $X, Y, Z$ » et ils peuvent en quelque sorte nous mener sur une autre voie parce que nous avons eu une interaction humaine.

Leila : Oui, un deuxième cerveau.

\section{Base de données et son contenu inconnus ou inaccessibles}

Manque de familiarité avec l'aspect de la base de données. Les personnes interrogées ne savaient pas exactement comment les entrées relatives aux éclosions étaient saisies dans la base de données ni quels champs et catégories étaient inclus.

Marie : Je suppose que ce que je veux dire, c'est que je plaide l'ignorance, tout ce que je sais, c'est ce qu'il y a (...) dans les rapports que j'ai reçus. 
La PAIFOD n'est pas accessible au public ni consultable. Interrogés sur un dictionnaire de données, les participants ont exprimé leur intérêt ou déclaré que chaque base de données devrait disposer d'un tel dictionnaire. De plus, ceux qui n'avaient pas demandé de rapports d'enquête sommaires sur les éclosions auparavant ne savaient pas non plus comment procéder ni même où trouver des informations sur la PAIFOD.

Rachel : Je pense qu'il serait bon que les gens sachent également ce qui est inclus dans la base de données et comment elle est utilisée de manière standard.

Inclusion ou modification des champs de données ou du processus de demande. Bien qu'elles ne connaissent pas toute l'étendue de la base de données, les personnes interrogées ont suggéré d'ajouter des champs (e.g., données spatiales, données génomiques, sexe des personnes atteintes, agents chimiques et physiques, points de vente communs) et se sont montrées ouvertes à l'idée de mettre en place un formulaire de demande standard.

Joon : Je préfère l'avoir et ne pas en avoir besoin que d'en avoir besoin et ne pas l'avoir.

Manon : Nous venons de découvrir que beaucoup de renseignements, je pense, dans ce champ appelé « Notes » ou quelque chose comme ça, n'étaient pas vraiment, il était difficile d'extraire des informations, en fait cela prenait beaucoup de temps.

\section{Demande d'une plateforme en ligne en libre accès}

Intérêt pour une interface infonuagique et intention de I'utiliser souvent. Les participants ont suggéré qu'une plateforme Web était la prochaine étape idéale pour la PAIFOD, car elle faciliterait l'accès, permettrait de générer des rapports et des graphiques personnalisables et faciliterait l'examen en cours de route des entrées d'éclosions.

Shelly : Je suis d'accord avec Luc et Rachel sur le fait que si elle est facilement accessible, elle sera probablement plus facile à utiliser.

Rebecca : Je suis également d'accord avec cela.

L'utilisation actuelle est limitée ou occasionnelle pour la plupart des clients. De nombreux clients n'étaient que des utilisateurs occasionnels. Ils ont déclaré qu'ils utiliseraient la PAIFOD plus souvent si elle était accessible en ligne sans avoir à passer par un « surveillant ».

Kim : Je pense qu'il serait plus facile et plus pratique que la base de données soit accessible aux chercheurs pour qu'ils puissent la consulter eux-mêmes. Par exemple, imaginez que vous deviez demander à quelqu'un de chercher dans PubMed à chaque fois pour vous au lieu de le faire vous-même.

Nécessité de disposer de sorties de données, de sorties graphiques et de formats de rapport flexibles. La plupart des utilisateurs préféreraient que les rapports soient fournis sous forme de feuilles de calcul Microsoft Excel plutôt qu'en PDF, ce qui est la norme actuelle. Par exemple :

Rose : Ce serait plus facile si les résultats étaient toujours affichés dans Excel.

Susan : J'aurais besoin d'une base Excel avec la capacité de filtrer, et j'aime bien Excel parce qu'on peut en quelque sorte faire le tir... directement dans un programme de statistiques.

L'élément humain peut être bénéfique pour guider les utilisateurs. Certains participants ont reconnu la valeur d'une interaction avec le gestionnaire de la base de données pour aider à produire les bons résultats.

Olivia : Peut-être un contact humain en cas de problèmes ou de questions. Une personne-ressource pour toute aide technique.

\section{Possibilité de ressource connue et couramment utilisée dans le domaine de la salubrité des aliments}

Ouverture à la collaboration. Les personnes interrogées ont suggéré que la collaboration permettrait d'améliorer le nombre d'éclosions saisies dans la PAIFOD, en particulier les foyers récents, ce qui renforcerait la base de données.

Moshe : dirais qu'à la base, il faut d'abord avoir une conversation avec nous...

Rachel : Je pense que nous pourrions simplement collaborer davantage les uns avec les autres à ce sujet. Cela pourrait nous aider à répondre à certains de nos besoins, probablement, et nous pourrions aussi répondre à certains de leurs besoins.

Il faut s'attaquer aux obstacles institutionnels. Les clients ont reconnu que certains obstacles institutionnels peuvent apparaître lorsqu'on essaie d'étendre la couverture de la PAIFOD.

Farid : Oui, le fait d'avoir des protocoles d'accord qui permettent le partage des données, en particulier lorsque des questions de salubrité des aliments sont en cause, peut aider un peu plus à la transparence de l'information et à l'instantanéité. Si la base de données propose un accès ouvert aux informations, c'est l'idéal..

Une ressource importante avec un potentiel d'expansion de l'utilisation au niveau international. Les utilisateurs ont vu beaucoup de potentiel dans la PAIFOD, car elle contient 
des renseignements sur les éclosions internationales d'origine alimentaire.

Moshe: Vous savez comme, "Oh vous avez, qu'est-ce qu'on en sait? Oh, ça va, la PAIFOD canadienne, oui, les Canadiens ont l'Agence de santé publique du Canada. "... si je la gérais, je verrais cela comme une évidence, toute occasion de valeur potentielle, de valeur organisationnelle, ensemble, qui la rend accessible à l'utilisateur...

\section{Discussion}

Les participants à l'étude étaient à l'aise dans l'utilisation des bases de données modernes comme celles de PulseNet Canada, qui est également utilisé pour cibler les éclosions de maladies d'origine alimentaire (11), et le National Outbreak Reporting System, accessible au public, qui signale toutes les éclosions de maladies d'origines hydriques, alimentaires et à entérobactéries connues des Centers for Disease Control and Prevention (CDC) (12).

Comme de plus en plus de bases de données sur la salubrité des aliments sont mises en ligne $(13,14)$, cette plateforme s'est avérée préférable pour les activités liées au travail, car l'accès peut être immédiat, les rapports peuvent être générés de manière flexible et la surveillance de la santé publique est plus rapide et plus réactive.

Les entretiens indiquent aussi clairement que les clients étaient satisfaits de l'ampleur des détails fournis par la PAIFOD, mais qu'ils aimeraient avoir accès à davantage de champs. Un nombre croissant de techniques et d'indicateurs scientifiques sont utilisés pour cerner les problèmes de salubrité des aliments et les agents pathogènes, dont certains permettent aux chercheurs d'effectuer des analyses approfondies dans le cadre de leurs travaux visant à protéger l'approvisionnement alimentaire canadien. Comme la PAIFOD rassemble des informations provenant de rapports accessibles au public, la base de données devrait envisager d'ajouter de nouveaux champs et catégories au fur et à mesure de la publication des rapports.

Une autre possibilité serait que cette base de données établisse des partenariats avec d'autres agences. En général, les systèmes de surveillance comme PulseNet Canada, PulseNet USA et la nouvelle initiative du gouvernement canadien, le Réseau canadien d'information sur la sécurité alimentaire, sont des répertoires de données partagées qui permettent aux organismes de réglementation locaux, d'état et provinciaux/ territoriaux et fédéraux d'accéder aux ressources et de les partager rapidement $(11,15,16)$. Toutefois, les informations contenues dans ces réseaux ne sont pas accessibles au public. La PAIFOD devrait viser à établir des liens qui permettront d'agrandir la base de données tout en la rendant accessible au public.
Pour la suite des choses, la PAIFOD devrait cibler une plateforme plus actualisée et plus conviviale, offrant ainsi un libre accès comme d'autres bases de données sur les épidémies qui ont fait leurs preuves; une flexibilité quant aux types de rapports générés; une plateforme plus complète qui comprend de nouveaux champs et catégories de données; une plus grande variété d'experts en sécurité alimentaire et d'épidémiologistes; et une plus grande collaboration entre les partenaires canadiens et internationaux pour améliorer la profondeur et promouvoir I'utilisation de la PAIFOD. L'expansion continue de la PAIFOD peut aider à révéler les tendances, à cibler les lacunes et à déterminer l'efficacité des futures interventions sur la réduction des maladies d'origine alimentaire.

\section{Limites}

La plupart des participants à cette évaluation étaient des employés du gouvernement fédéral. Bien qu'ils semblent être les principaux utilisateurs de la base de données, leurs besoins peuvent différer de ceux d'autres clients, ce qui pourrait avoir eu une incidence sur les thèmes générés.

Deuxièmement, il n'était pas clair si les entretiens de groupe contenaient des réponses homogènes parce que les participants provenaient du même service ou en raison des structures de pouvoir existantes (17). Les enquêteurs ont observé que les opinions étaient disproportionnellement plus nombreuses parmi les personnes ayant une plus grande expérience des bases de données et celles occupant un poste de direction. L'absence de désaccords au sein des groupes indique qu'il pourrait être bénéfique de mener les évaluations futures exclusivement par l'entremise d'entretiens individuels.

\section{Conclusion}

Cette évaluation du programme a exploré les expériences actuelles des utilisateurs de la PAIFOD, y compris l'étendue des connaissances sur la base de données des éclosions, ses forces, ses faiblesses et les domaines à améliorer grâce à une approche d'analyse thématique qualitative. Dans l'ensemble, la plupart des intervenants ne connaissaient pas la totalité du contenu de la base de données parce qu'ils ne recevaient que des rapports de synthèse; les utilisateurs actuels et précédents estimaient que la base de données était un outil utile qui les aidait dans leurs activités sur la salubrité des aliments; et presque tous les répondants étaient intéressés par une plateforme en ligne à accès libre et estimaient que la PAIFOD était une ressource précieuse et unique qui avait un potentiel d'expansion. Les personnes interrogées ont recommandé des améliorations à la base de données afin d'améliorer leur utilisation personnelle, ainsi que la légitimité et la réputation de la PAIFOD.

Les nombreuses conclusions de cette étude étaient vastes et pouvaient être appliquées à d'autres bases de données de surveillance des maladies infectieuses et d'origine alimentaire. 


\section{Déclaration des auteurs}

I. Y. - Conceptualization, funding acquisition, analysis, investigation, methodology, project administration, resources, software, supervision, validation, writing-review \& editing A. T. - Analyse, enquête, méthodologie, administration du projet, validation, rédaction du projet original

M. M. - Conceptualisation, ressources, validation, rédactionrévision et édition

L. W. - Conceptualisation, ressources, validation, rédactionrévision et édition

\section{Intérêts concurrents}

Aucun.

\section{Remerciements}

Les auteurs remercient les participants qui ont donné de leur temps pour apporter une contribution précieuse à la Base de données publique internationale sur les éclosions des maladies d'origine alimentaire (PAIFOD), D. Ayache pour avoir fourni des données de synthèse sur le contenu de la PAIFOD et J. Greig pour ses nombreuses années consacrées au développement, à l'actualisation et à la coordination de la PAIFOD.

Merci également à la gestionnaire de la PAIFOD, M. Mascarenhas, que l'on peut joindre à l'adresse mariola. mascarenhas@canada.ca.

\section{Financement}

Cette recherche a été soutenue par un financement du Laboratoire national de microbiologie de l'Agence de la santé publique du Canada.

\section{Références}

1. World Health Organization. Foodborne disease outbreaks: guidelines for investigation and control. Geneva $(\mathrm{CH})$ : WHO; 2008. https://www.who.int/foodsafety/publications/ foodborne_disease/outbreak_guidelines.pdf

2. Ganz K, Yamamoto E, Hardie K, Hum C, Hussein H, Locas A Steele M. Microbial safety of cheese in Canada. Int J Food Microbiol 2020;321:108521. DOI PubMed

3. Greig JD, Ravel A. Analysis of foodborne outbreak data reported internationally for source attribution. Int J Food Microbiol 2009;130(2):77-87. DOI PubMed

4. Nasheri N, Vester A, Petronella N. Foodborne viral outbreaks associated with frozen produce. Epidemiol Infect 2019;147:e291. DOI PubMed
5. Wilhelm B, Fazil A, Rajić A, Houde A, McEwen SA. Risk profile of hepatitis $E$ virus from pigs or pork in Canada. Transbound Emerg Dis 2017;64(6):1694-708. DOI PubMed

6. Conseil de recherches en sciences humaines Conseil de recherches en sciences naturelles et en génie du Canada Instituts de recherche en santé du Canada. Énoncé de politique des trois conseils : Éthique de la recherche avec des êtres humains - EPTC 2 (2018). Ottawa (ON) : Gouvernement du Canada; 2018 (accédé 2020-02-12). https://ethics.gc.ca/fra/policy-politique_tcps2-eptc2_2018. html

7. Carter N, Bryant-Lukosius D, DiCenso A, Blythe J, Neville AJ. The use of triangulation in qualitative research. Oncol Nurs Forum 2014;41(5):545-7. DOl PubMed

8. Guba EG, Lincoln YS. Fourth generation evaluation. Los Angeles (CA): Sage Publications; 1989. https://catalogue.nla. gov.au/Record/1153062

9. Burr V. Social constructionism (3rd ed.). London: Taylor and Francis Inc.; 2015. DOI

10. Braun V, Clarke V. Using thematic analysis in psychology. Qual Res Psychol 2006;3(2):77-101. DOI

11. Agence de la santé publique du Canada. PulseNet Canada. Ottawa (ON) : Gouvernement du Canada; 2019 (accédé 2020-03-18). https://www.canada.ca/fr/sante-publique/ programmes/pulsenet-canada.html

12. Centers for Disease Control and Prevention. National Outbreak Reporting System (NORS). Atlanta (GA): CDC 2019 (accessed 2020-03-18). https://www.cdc.gov/nors/ index.html

13. Stevens EL, Timme R, Brown EW, Allard MW, Strain E, Bunning K, Musser S. The public health impact of a publically available, environmental database of microbial genomes. Front Microbiol 2017;8:808. DOI PubMed

14. Taboada EN, Graham MR, Carriço JA, Van Domselaar G. Food safety in the age of next generation sequencing, bioinformatics, and open data access. Front Microbiol 2017;8:909. DOI PubMed

15. Agence canadienne d'inspection des aliments. Réseau canadien d'information sur la salubrité des aliments (RCISA). Ottawa (ON) : Gouvernement du Canada; 2018 (accédé 2020-03-18). https://www. inspection.gc.ca/les-sciences-et-les-recherches/rcisa/ fra/1525378586176/1525378959647

16. Tolar B, Joseph LA, Schroeder MN, Stroika S, Ribot EM, Hise KB, Gerner-Smidt P. An overview of PulseNet USA databases. Foodborne Pathog Dis 2019;16(7):457-62. DOI PubMed

17. Smithson J. Using and analysing focus groups: limitations and possibilities. Int J Soc Res Methodol 2000;3(2):103-19. $\mathrm{DOI}$ 